Rhode Island College

Digital Commons @ RIC

$1-1-2014$

\title{
Preventing Ventilator-Associated Pneumonia: Educating Emergency Room Nurses
}

Dana M. Ferrazzano

Rhode Island College

Follow this and additional works at: https://digitalcommons.ric.edu/etd

Part of the Other Education Commons, and the Other Nursing Commons

\section{Recommended Citation}

Ferrazzano, Dana M., "Preventing Ventilator-Associated Pneumonia: Educating Emergency Room Nurses" (2014). Master's Theses, Dissertations, Graduate Research and Major Papers Overview. 233.

https://digitalcommons.ric.edu/etd/233

This Major Paper is brought to you for free and open access by the Master's Theses, Dissertations, Graduate Research and Major Papers at Digital Commons @ RIC. It has been accepted for inclusion in Master's Theses, Dissertations, Graduate Research and Major Papers Overview by an authorized administrator of Digital Commons @ RIC. For more information, please contact digitalcommons@ric.edu. 
PREVENTING VENTILATOR-ASSOCIATED PNEUMONIA: EDUCATING EMERGENCY ROOM NURSES

by

Dana Ferrazzano

A Major Paper Submitted in Partial Fulfillment of the Requirements for the Degree of

Master of Science of Nursing

in

The School of Nursing

Rhode Island College

2014 


\begin{abstract}
Ventilator-associated pneumonia (VAP) is the second most common hospital acquired infection and is primarily found in the intensive care units. The presence of an endotracheal tube is the primary risk factor for developing VAP. Many times the process of intubation occurs pre-hospital, in the emergency room (ER) or in the operating room. Ventilator associated pneumonia is associated with increased hospital stay and costs. Evidence-based guidelines have been developed to decrease the occurrence of VAP and decrease patient mortality. Preventative measures are initiated on the intensive care unit once the patient is transferred. The purpose of this study was to evaluate the impact of an educational program on ER nurses knowledge of VAP and preventative measures to decrease VAP.
\end{abstract}

The study took place at Rhode Island Hospital, a level 1 trauma center located in Providence, RI. Participants were ER nurses who worked in the critical care area of the ER. A pre-intervention-posttest design was used to evaluate emergency room nurses knowledge regarding VAP. The intervention consisted of a posterboard and study guide that provided information regarding evidence-based guidelines that could be implemented in the ER such as oral care, and head of bed elevation. The overall results on the pre and posttest demonstrated that ER nurses were unaware that oral care and the use of chlorhexidine were important interventions to reduce the incidence of developing VAP. Recommendations and implications for advanced practice nursing and the need for future research are discussed.

\title{
Table of Contents
}

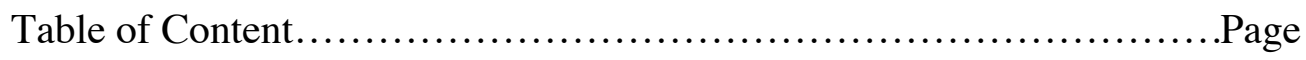

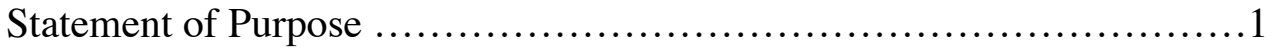




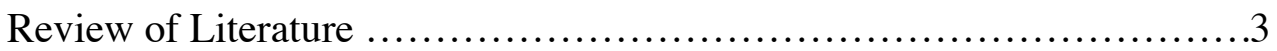

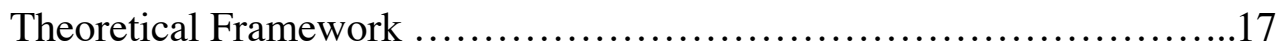

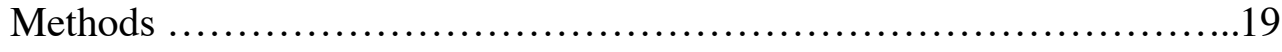

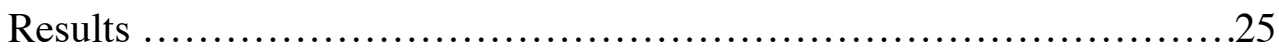

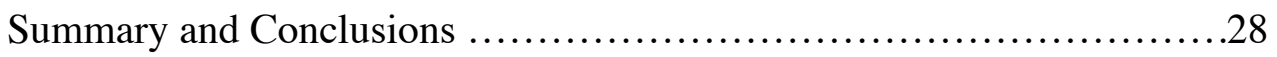

Implications and Recommendations for Advanced Nursing Practice ......33

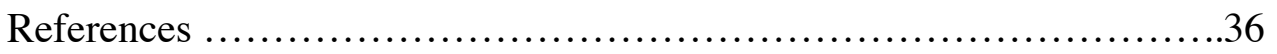

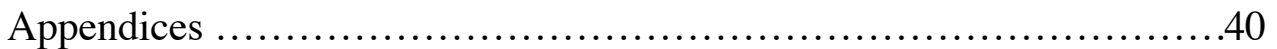


Preventing Ventilator-Associated Pneumonia:

Educating Emergency Room Nurses

\section{Problem Statement}

Healthcare-associated infections (HAIs) represent a huge financial burden and contribute significantly to health-care costs. Average medical costs across hospitals in the United States (US) due to HAIs vary from 28.4 to 33.8 billion dollars (Scott, 2009). The Centers for Disease Control and Prevention (CDC, 2010) defined HAIs as infections that patients acquire during hospitalization while receiving treatment for healthcare conditions. There are five identified HAIs identified by the CDC (2010), including central line associated bloodstream infections (CLASBI), catheter- associated urinary tract infection (CAUTI), surgical site infection (SSI), clostridium difficile infection (CDAD), and ventilator-associated pneumonia (VAP).

Ventilator associated pneumonia is the most common infection in mechanically ventilated patients and the second most common HAI (Sedwick, Lance-Smith, Reeder, \& Nardi, 2012). The mortality rate for VAP ranges between 20 and $70 \%$ and exceeds those of other HAIs (Mietto, Pincoroli, Patel, \& Burra, 2013). Sedwick et al. (2012) reported that The Center for Medicare and Medicaid Services (CMS) recently listed VAP as one of the "reasonably preventable diseases" leading to increased morbidity, mortality, and health care costs. Increased length of stay associated with VAP ranges from four to 13 days and results in increased hospital costs of approximately $\$ 40,000$ per patient (Gallagher, 2012).

Nurses have a direct impact on patient care outcomes in regard to HAIs, including VAP, and lack of current and evidence-based knowledge among nurses regarding preventative measures contributes to increased incidence (Gallagher, 2012). The Institute for Healthcare Improvement (IHI) (Gallagher) implemented a ventilator bundle that consisted of six interventions aimed to reduce the incidence of VAP, 
including daily oral care with $0.12 \%$ chlorhexidine, head of bed elevation 30 to 40 degrees, subglottal suctioning, administration of proton pump inhibitors or histamine blockers, daily sedation vacation, and deep vein thrombosis prophylaxis. Education of nurses regarding these interventions has been shown to be effective in reducing the incidence of VAP (Blot, Labeau, Vandijck, Aken, \& Claes, 2007).

Increased length of time in the ER may contribute to the delay in starting preventative measure in mechanically ventilated patients. The role of ER nurses is to treat, stabilize, and move the patient to the appropriate level of care, yet many evidencebased guidelines are initiated in the ER prior to transfer to the appropriate level of care. Currently, little literature exists regarding early initiation of VAP prevention beginning in the emergency room. This researcher conversed with several ER nurses and identified a lack of knowledge regarding VAP and preventative measures. Nurses expressed interest in learning about VAP and preventative measures with the goal of implementing the IHI VAP bundle in the ER. The purpose of this study was to evaluate the impact of an educational program on ER nurses' knowledge of VAP and preventative measures to decrease VAP.

\section{Review of Literature}

A literature review was conducted using the following databases and websites: PUBMED; CINAHL; Proquest; Up-to Date; CDC; IHI; and CMS. Keywords included ventilator associated pneumonia and critical care, emergency room and ventilator associated pneumonia, VAP and preventative measures, nurses' knowledge and VAP, evidence-based guidelines and VAP. Articles searched and reviewed were published within the last 10 years. 


\section{Introduction}

Health care costs in the United States (US) have been the center of discussion for many years. Healthcare-associated infections are a source of increased material and human cost in the US and evidence-based guidelines and protocols have been developed in the attempt to decrease the incidence of HAIs. Ventilator associated pneumonia is a HAI that occurs primarily in intubated and mechanically ventilated patients. The prevalence rate of VAP is controversial due to the lack of standardized diagnostic criteria and definitions (Grgurich, Hudcova, Lei, Sarwar, \& Craven, 2013). Ventilator associated pneumonia has been identified as the second most common HAI (Grap, Munro, Unoki, Hamilton, \& Ward, 2012) and adds an additional $\$ 40,000$ to overall hospital costs secondary to the increased length of stay and treatment. The occurrence rate averages at $10-20 \%$ in intubated patients receiving mechanical ventilation (Grap et al.). Ventilator associated pneumonia is categorized as early or late onset: early onset occurs within 4872 hours after intubation, with late onset occurring after 72 hours (Amin, 2009). The average time to diagnosis is approximately three days (Grap et al., 2012).

Although published guidelines to prevent VAP are available, nurses' lack of knowledge related to the guidelines may contribute to non-adherence to the guidelines (Meherali, Parpio, Ali, \& Javed, 2011). Nurses have a direct impact on the incidence of HAIs overall and VAP in particular, and are able to perform recommended nonpharmacologic interventions independently (Gallagher, 2012). Interventions such as oral care, head of bed elevation, and suctioning are daily tasks that should be performed regularly on ventilator patients. Many times nurses perform these measures and are unaware of the scientific reasoning; providing education to nurses empowers them to provide optimal care to their patients. Adherence to institutional protocols for VAP prevention can significantly impact the incidence of it (Sedwick et al., 2012).

\section{Pathophysiology of VAP Development}


Ventilator associated pneumonia is a HAI that is caused by the presence of an endotracheal tube (ETT) (Mietto et al., 2013). An ETT is placed either pre-hospital, during surgery, or in the ER, to assist with airway maintenance. Intubation is an invasive procedure, and puts patients at risk in developing a HAI (Mietto et al.). The process of intubation decreases the body's natural response to infection by disrupting the ability to initiate a cough or gag reflex that assists in expelling secretions (Sedwick et al., 2012) As a result, secretions settle around the posterior portion of the pharynx and can eventually lead to micro-aspiration (Sedwick et al.). Proper inflation of the ETT cuff does not prevent micro-aspiration, because there is a small opening that allows leakage of the secretions to travel (Mietto et al., 2013)

Mietto et al. (2013) identified that the polyvinyl surface of ETTs serves as secondary reservoir for bacterial colonization, referred to as biofilm. Biofilm is defined as a cluster of microorganisms clumped together within a substance made of polysaccharides, proteins, and DNA that forms a mechanical scaffold around bacteria (Mietto et al.). This develops rapidly within hours of intubation. Suctioning and positive pressure from mechanical ventilation cause the bacteria to detach from the ETT and travel to the lower respiratory tract (Mietto et. al.). Offending pathogens that cause VAP include aerobic gram-negative bacilli Staphylococcus aureus, Enterococci, Enterobacteraceae, Pseudomonas aeruginosa , Acinetobacter baumannii and Candida albicans. These organisms are identified by culture from the ETT and tracheal secretions (Meherali et al., 2011). Pseudomonas and Acinetobacter are two highly infectious pathogens that are associated with an increased mortality rate among intubated patients (Amin, 2009). Early diagnosis and treatment of VAP decreases patient morbidity and mortality, and length of stay (Amin).

\section{Diagnosis of VAP}


Reduction of VAP is a national patient safety goal (Mietto et al., 2013). Currently, there is no gold standard for diagnosis of VAP, and as a result multiple definitions and criteria have been used throughout the years (Grgurich et al., 2013). Lack of a gold standard for diagnosis creates variability in VAP rates among health care institutions (Grgurich et al.). The recommended criterion from the CDC (2013) consists of clinical presentation, laboratory, and diagnostic results that are identified in the Pneumonia Flow Diagram (Appendix A).

Ventilator-associated pneumonia rates vary based on the criteria used to make a diagnosis. Clinical criteria are based on two positive findings from the following signs and symptoms: fever $>38$ degrees; leukocytosis or leukopenia (increased white blood cell count, or low white blood cell count); and purulent (infectious) secretions combined with results of chest xray (Grgurich et al.). Microbiology assists in the diagnosis of VAP based on findings obtained from a broncho-alveolar lavage or protected specimen brush. New consolidation or infiltrates that were not present prior to intubation are part of the diagnostic criteria to confirm VAP. Chest x-rays lack specificity because certain conditions such as pulmonary embolism, congestive heart failure, and atelectasis (decrease expansion of alveoli) can mimic an infiltrate or consolidation (Grgurich et al). The CDC (2013) is currently working towards changing surveillance definitions for VAP. The following literature explores the difference in diagnostic criteria currently used among different institutions and intensive care units.

Rea-Neto, Youseff, Tuche, Brunkhorst, Ranieri, Reinhart, and Sakr (2008) performed a qualitative systematic review of the literature regarding the diagnosis of VAP. Sixty-four articles published between January 1966 and June 2007 met the inclusion criteria. The purpose of the study was to compare various criteria for diagnosing VAP in the intensive care unit. The focus was on the value of clinical diagnosis, microbiological culture techniques, and biomarkers of host response. The 
investigators looked at clinical criteria, bacteriologic data, and quantitative cultures to assess the accuracy of VAP diagnosis. The conclusion of the systematic review was that there was not any one tool used that definitively diagnosed VAP.

Systemic inflammatory response syndrome (SIRS) is a method used by some intensive care units to identify VAP (Novosel et al., 2012). Criteria for systemic inflammatory response syndrome includes temperature greater than 38 degrees or less than 36 degrees, heart rate greater than 90 beats per minute, respiratory rate greater than 20 breaths per minute, $\mathrm{PaCO} 2$ less than $32 \mathrm{mmHg}$, and white blood cell count greater than 12,000 cells $/ \mathrm{mm}$ or less than 4,000 cells $/ \mathrm{mm}$. Another criteria used is the Clinical Pulmonary Infection Score (CPIS), which is based on six criteria: fever; leukocytosis; tracheal aspirates; oxygenation; radiographic infiltrate: and semi-quantitative cultures of tracheal aspirates with Gram stain (Neto et al., 2008).

A retrospective evaluation using a database of mechanically ventilated patients was performed at a level 1 trauma center in Virginia between January 2010 and June 2011 (Novosel et al., 2012). The purpose of the study was to assess the different criteria to diagnosis VAP used by the trauma service and the infection control committee. The trauma service identified VAP using the systemic inflammatory response syndrome. The infection control committee diagnosed VAP based on the National Nosocomial Infection Surveillance (NNIS) criteria. The findings of the study revealed that using the NNIS standard led to under-reporting of VAP. Limitations of this study included that is was completed in a single institution, VAP rates were not calculated in per 1000 ventilator days, and standard x-rays were not performed which is part of the NNIS algorithm.

In summary, there is no gold standard available for the diagnosis of VAP but there are many diagnostic tools available to assist with diagnosis. The clinical diagnosis of VAP leads to the use of increased antibiotic therapy and can lead to multi-resistance drug therapy. In patients with high suspicion of VAP based on clinical findings, further 
workup with diagnostic testing and cultures should be performed to assist with confirmation of diagnosis. Treatment with antibiotics should begin immediately when there is a suspicion of VAP, as delays in antibiotic administration can lead to increased mortality (Neto et al., 2008).

\section{Emergency Room and VAP}

Critical care interventions start in the ER and evidence-based protocols for a variety of conditions are in place to care for these emergent patient. When an early intervention is initiated, it has been shown to decrease mortality and the length of an ICU stay (Grap et al., 2012). Grap et al. reviewed an article regarding the use of evidencebased guidelines to reduce VAP, the goal of which was to evaluate the need for early VAP intervention in the ER. Grap et al. identified the need to initiate some interventions in the ER, including. head of bed elevation to decrease aspiration, oral care with use of chlorhexidine (gel or mouth rinse), management of ETT cuff pressure, subglottic suctioning, and ulcer prophylaxis. There is evidence to support that early goal directed therapy initiated in the emergency room has a direct impact on patient mortality (ChungEsaki, Wilson, \& Rodriguez, 2011) but there is no research to support if early interventions in the emergency room decrease the incidence of VAP.

Trauma patients have been identified as high-risk patients for VAP. Typically, trauma patients are intubated emergently in the pre-hospital setting or the emergency room. Carr et al. (2007) completed a retrospective case-control study of trauma patients and risk of VAP. The study was conducted at a 650 bed level 1 trauma center in Southwest Philadelphia. The purpose of the study was to evaluate if increased length of time in the emergency room increased the risk for VAP in trauma patients. Ventilatorassociated pneumonia was identified in patients who had a fever greater than 38 degrees Celsius, leukocytosis, positive sputum gram stain, and chest $\mathrm{x}$-ray confirming new infiltrate. Subjects included in the study had experienced blunt trauma that required 
emergent intubation $(\mathrm{N}=140)$. Demographics and clinical data collected included age and gender, injury severity score, chest abbreviated injury score, and head abbreviated injury score. Emergency room length of stay was compared between patients who developed VAP versus patients who did not. Length of stay in the ER was found to be a risk factor of VAP development (OR 1.21, p<0.05, 95\% CI- 1.04-1.39). Longer intensive care unit stays (16.3 vs. $5.1 \mathrm{p}<0.001)$ and longer hospital stays (25.2 vs. 11.2, p<0.0001) were associated with VAP. Every hour spent in the emergency room increased a patient's probability of developing VAP by approximately $20 \%$. Further research is needed regarding the impact of ER length of stay of intubated patients and the risk for VAP.

\section{Evidence-Based Guidelines}

Evidence-based guidelines were developed to decrease HAI rates, provide optimal care to patient using the best research available, and optimize patient outcomes. The Institute of Healthcare Improvement (IHI) (Gallagher, 2012) developed a ventilator bundle that originally consisted of four evidence based interventions that have been proven to decrease the incidence of VAP and other HAIs: head of bed (HOB) elevation to 30 degrees or more; daily sedation vacation; weaning assessment; and ulcer and deep vein thrombosis prophylaxis (Bonten, 2011). Oral care with chlorhexidine was recently added since dental plaque contributes to increased risk for VAP (Bonten). Other measures incorporated into the bundle are subglottic suctioning, hand-washing protocol, and a feedback tool for staff (Sedwick et al., 2012). Since VAP is a reportable condition, The Joint Commission requires hospitals to prove they have preventative guidelines in place to reduce HAIs (Gallagher). Health care institutions have adopted various versions of the bundle based on their protocols, but due to the increased cost of special equipment, i.e. subglottic suctioning, many facilities have not adapted the complete bundle.

A retrospective study was conducted by Gallagher from August and September 2010 (control group) and August through September 2011 (experimental group) (2012) 
with the purpose of measuring VAP rates after introducing ventilator bundle.

Interventions implemented as part of the study protocol included hand washing, HOB elevation to 30 degrees, checking ventilator tubing for condensation, daily breathing trials, sedation vacation, DVT and ulcer prophylaxis, and oral care every four hours with chlorhexidine gluconate $0.12 \%$. Ventilator associated pneumonia was identified as a new consolidation on chest $\mathrm{x}$-ray in combination with temperature greater than 38.3 degrees Celsius, white blood cell count greater than 10,000, and an identified pathogen from bronchial lavage. Thirty-eight patients were part of the experimental group and received interventions from the ventilator bundle, and 45 patients were assigned to control group, which received usual care. An educational intervention for nurses was implemented which consisted of online learning with the ability to obtain continuing education credits. The experimental group demonstrated reduced levels of VAP in comparison to the control group ( 0 cases per 1000 ventilator days vs. 25.5 cases per 1000 ventilator days). Study limitations included lack of randomization and small sample size. The study demonstrated that incorporating a bundle along with focused staff education can impact VAP rates.

Sedwick et al. (2012) performed a chart audit between January and December of 2009 in a Philadelphia hospital intensive care unit. The purpose of this study was to identify if implementation of ventilator bundles decreased incidence of VAP. Nurses, physicians, and respiratory therapists were involved in implementing the program. A goal was set among the healthcare team to achieve a zero rate and $100 \%$ bundle compliance with in a year. The intervention bundle included a hand-washing protocol, HOB alarms, DVT and ulcer prophylaxis, sedation vacation and weaning, subglottic suctioning and oral care. Education was provided on each aspect of the bundle and VAP bundle informational sheets were dispersed to nursing staff. Despite the education, nursing compliance with the intervention bundle did not increase. The research team then placed 
programs on computers to provide nurses with feedback regarding compliance and VAP rates. Subseqently, compliance rates increased after nurses were able to associate compliance with outcomes. Overall results revealed a decrease in VAP rates from 4964 ventilator days (9.47 cases per 1000 ventilator days) to 4709 ventilator days (1.9 cases per 1000 ventilator days). Oral care was alternated between respiratory therapy and nursing, requiring oral care every two hours and use of chlorhexidine twice a day. Oral care never reached $100 \%$ compliance despite the fact that oral care has been shown to be effective in reducing VAP rates (Sedwick et al.). Evidence-based guidelines are only effective if there is increased compliance with interventions being used.

\section{Assessment of Effect of Education on Nurses' Knowledge}

Many studies have been performed to assess the impact of an educational program regarding VAP prevention for nurses and VAP rates. Most of the ventilator bundle can be implemented and managed by nursing. Nurses may lack knowledge regarding preventative measures and/or may be non-compliant in adhering to preventative guidelines. Assessing nurses' knowledge of preventative measures is the first step in an educational intervention. Most of the studies reviewed involved administering a pretest to examine nurses' knowledge, implementing a planned educational intervention, and administering a post-test to assess the effectiveness of interventions (Meherali et al., 2011).

Evaluation of nurses' knowledge was completed in two separate studies that involved a multiple-choice questionnaire developed by the researcher and validated for reliability. The purpose of these studies was to determine nurses' knowledge regarding evidence-based guidelines for VAP prevention. Both of the studies used the same questionnaire developed by Blot et al (2007). Content consisted of knowledge regarding preferred route of intubation, frequency of ventilator circuit changes, airway humidifiers, frequency of humidifier changes, preferred suction units, open or closed, frequency of 
suction catheter change, type of bed preferred, patient positioning, and influence of an extra lumen on ETT for subglottic secretion drainage (Blot et al., 2007). Blot et al. administered and collected the questionnaire at the annual congress of the Flemish Society for Critical Care Nurses. Six hundred and thirty eight nurses completed the questionnaire out of 855 participants. Demographic data collected was based on gender, years of intensive care experience, number of hospital beds, and any certification held by the respondent. Results of the demographic data showed that $74 \%(n=472)$ of the nurses were female, $24 \%(\mathrm{n}=153)$ had less than one year of intensive care experience, $17.4 \%(\mathrm{n}$ $=111)$ had 1-5 years of experience, $15.7 \%(\mathrm{n}=100)$ had 6-10 years of experience, and $43.0 \%(n=274)$ had greater than 10 years of experience. Respondents with degrees held in emergency or critical care experience averaged $68 \%$, or approximately 437 nurses. The questions were worth one point for every correct answer and the average score was 3.7 out a possible 9 points $(41.2 \%)$. Overall, the resulting low scores demonstrated the need for education regarding VAP prevention. A major strength of the study was the large sample size; a limitation was that it was restricted to one geographic location. Selection biases identified in the study were that nurses who attended the conference may have had more skills or motivation and may have attended only to obtain for education units to maintain their degree.

The Blot et al. (2007) questionnaire was used in a study performed by Labeau, Vandijck, Rello, Rosa, Wenisch, and Backman et al. (2008). The survey was dispersed via mail to nurses in 22 European countries between October 2006 and March 2007. Demographics collected were similar to the Blot et al. study with the addition of nationality. Three hundred and twenty nine questionnaires were collected from participants. The average score was 4.9 out of possible 9 points, with the more experienced nurses achieving the highest scores. Lower scores were found among nurses working in a large intensive care unit. Limitations of the study included that preventative 
measures varied between local and national guidelines. Identified strengths of the study were that it encompassed a larger geographical region and sample size. In summary, the two studies demonstrated that nurses' knowledge was low despite whether the proposed guidelines differed among institutions or countries. Increased knowledge may not increase adherence to guidelines but lack of knowledge can contribute to non-adherence.

Meharali et al. (2011) used a pre-post test design on an 11 bed mixed medicalsurgical intensive care unit at a teaching hospital in Pakistan. The purpose of the study was to assess critical care nurses' knowledge regarding VAP. Forty nurses participated after they met inclusion criteria of having one or more years of experience in critical care. The pretest was a 10 question multiple-choice exam based on preventative guidelines. After completion of the pre-test, nurses participated in a five-hour educational session focused on management of VAP and evidence-based guidelines. The posttest was administered immediately after the session and at four weeks post. Posttest \#1 showed an increase in mean scores (pretest 7.8 + 2.9; posttest \#1 10.8 to 2.0). Scores on posttest \#2 administered four weeks later were lower $(9.8+2.1 ; \mathrm{p}=0.0001)$. Although, the scores in posttest \#2 were lower than posttest \#1, they were higher than the pretest scores. The pretest scores demonstrated a lack of knowledge regarding evidence-based guidelines of VAP prevention. Since the study occurred over just four weeks, there was not enough time to observe if VAP rates increased or decreased.

Gatell et al. (2012) completed a prospective, quasi-experimental, pre- and posttest design on a 16 bed medical/surgical unit. The purpose of the study was to assess the impact of education on nurses' knowledge of VAP and preventative measures. The study was conducted in three phases, including pre-intervention, intervention, and postintervention. The pre- and post-intervention phase consisted of a 22 multiple-choice test on non-pharmacological interventions in five categories: endotracheal aspiration; controlling gastric reflux; micro-aspiration prevention; oral hygiene; and documenting 
placement of ETT. Part of the pretest involved observing nurses at the bedside to monitor adherence to guidelines; nurses were aware of being observed but were not aware of the content or purpose of the observation. Educational sessions regarding VAP preventive measures, epidemiology, risk factors, and endotracheal secretion aspiration procedure were part of the intervention phase. Multiple sessions were held to ensure maximum attendance of the staff. Extracurricular material such as poster boards and leaflets were placed throughout the unit to strengthen the education provided. Guideline adherence increased $(\mathrm{p}=0.001)$ in using narrowest nasogastric tube, head of bed positioning, decrease in aspiration, correct ETT cuff pressure, using chlorhexidine during oral care, and documenting tube placement. Ventilator associated pneumonia rates did not significantly change (9.9 vs. 9.3 episodes/1000 days under mechanical ventilation), although there was a lower rate in the development of late onset VAP (>4 days) (4-6 vs . 3.1 episodes/1000 ventilation days, $\mathrm{p}=0.36$ ). Results also showed that increased workload effects adherence to guidelines because of nurses' performing more important tasks. When workload is increased nurses will not perform measures that they regard as less important such as nasogastric tube replacement, or completing charts.

The common theme of these studies is nurses' lack of knowledge regarding VAP and preventative measures. Educational sessions increased nurses' knowledge regarding VAP, though in some cases overall knowledge remained low. In some cases, education increased adherence to guidelines, but constant reinforcement and adequate staffing are important influencing variables. Increased knowledge and adherence did not significantly decrease VAP rates in some studies. Further controlled studies with larger samples over longer period of time are needed.

The purpose of the proposed study was to evaluate the impact of an educational program on ER nurses' knowledge of VAP and preventative measures to decrease VAP. 


\section{Theoretical Framework}

Malcolm Knowles' Adult Learning Theory was the theoretical framework used to guide this program development project. Knowles' theory is based on six principles: the need to know; the learners' self-concept; the role of the learners' experience; readiness to know; orientation to learning; and motivation (Knowles, Holtron, \& Swanson, 2005). The need to know is based on the learner's perception of why certain information is important to learn and is the starting point for all learning. This relates to the readiness to know and motivation to learn. When there is a need to learn and a benefit to learning, then adults will be more willing and successful in the learning environment. The learner's selfconcept and experience intertwine because the adult learner wants to be viewed by others and themselves as being self-directed, and their experience provides them with selfidentity. These principles appeal to a person's self-esteem and how the person is viewed by family, friends, and coworkers. Experience also provides greater resources for learning such as simulation exercises, group discussions, and problem solving techniques. Adults generally are ready learners; most learn because they are interested in and motivated to enhance their knowledge base, either for personal motivation to learn more or for professional status/career enhancement (Knowles et al.).

Nurses are adult learners; they are motivated to learn new material that will optimize and improve patient outcomes, and expand their knowledge base. When there is a lack of knowledge or experience regarding for example, certain medications or conditions, nurses will typically obtain information through multiple readily available resources. The effective learner uses these various sources in the form of experience, text, instruction, observation, or any other means to form a complete system of learning.

Educators must also acknowledge the adult learner concept that adult learners do not like to be mandated to learn (Knowles et al.). When education is mandated, it can 
lead to resistance in learning and lack of retaining the information. Educators should appeal to the learners' desire to learn and need for the information.

Reducing the incidence of ventilator associated pneumonia through initiating preventive measures in the emergency room is of interest to nurses in the emergency department as identified in preliminary interviews. A study guide and posterboard were readily accessible to ED nurses in the study group. These materials were developed with careful consideration of Knowles' adult learning principles. Nurses identified the need and interest in learning this content. The content is presented so that nurses can review it at their leisure in two different venues to enhance visibility, access and interest in learning. Using Knowles' Adult Learning Theory framework, it was anticipated that nurses would actively participate in this self -directed program. 


\section{Methodology}

\section{Purpose}

The purpose of this study was to evaluate the impact of an educational program on emergency room (ER) nurses' knowledge of ventilator-associated pneumonia (VAP) and preventative measures to decrease VAP.

\section{Design}

The design of the study was a non-probability, purposive, pre-, intervention, postintervention design.

\section{Sample/Site}

The sampling method used was purposive, nonprobability sampling. The subjects were nurses who worked in the critical care area of the Rhode Island Hospital (RIH) ER. Rhode Island Hospital is a 719- bed level one trauma center located in Providence, RI. The total potential sample size was approximately 130 critical care nurses. The inclusion criterion consisted of nurses working in the critical care area of the RIH ER. The exclusion criterion included traveling nurses, nurse practitioners, nurses who did not work in critical care, and all other non-nursing personnel.

\section{Development of the Educational Intervention}

The W.F Kellogg Foundation Logic Model (Kellogg Foundation, 2004) was used as the framework to assist in this program development. The goal in using the Logic Model was to assist the researcher in recognizing each component needed to achieve success of a program. The Logic Model consists of a template that includes: resources; activities; outputs; short and long term outcomes; and impact. Each of these steps guided the researcher to accomplish the goal of the program development (Appendix B).

Resources refer to the needs identified that will assist in development of the program. Resources may include human, financial, or organizational resources that will 
assist in directing the research. Identified resources included the Director of Infection Control and Clinical Nurse Specialist (CNS) in the ER.

A focused needs assessment was conducted. The Director of Infection Control supported the project as a means to potentially decrease VAP rates. The CNS in the ER encouraged education regarding VAP when aware that there were some preventative measures that could be started in the ER, such as mouth care and head of the bed elevation. During a discussion with ER nurses, it was identified that there was a lack of knowledge regarding what VAP is and how it can be prevented. Emergency room nurses treat and stabilize patients', and may not have the forethought of what happens to the patient once they are transferred to the appropriate unit.

Finally, the lack of literature regarding ER nurses and VAP prevention validated the need for this project. The literature supported the need for an educational intervention, based on the lack of research regarding early intervention in the ER. This was especially important since the literature also supported that early initiatives using evidence-based guidelines had the potential to decrease morbidity and mortality related to VAP.

Activities/Procedures refer to what is needed to tackle the problem at hand. Activities include processes, tools, or technology that are developed to implement the program. In order to accomplish the goal of the research project, the researcher developed educational materials that included a poster board and study guide (Appendix C), which were developed from the literature review, clinical experience, and available hospital resources. This researcher obtained the current preventative measures used by RIH and integrated them into the study guide and on the poster board. The poster board and study guide were also developed based principles derived from Knowles' Adult Learning Theory. Adults learn will learn new material at their time and pace. With this concept in mind, the researcher developed the poster board and study guide with the intent of allowing nurses to read the material at their own pace and at a time that was 
conducive to them. The ER is a fast paced environment and time is limited for nurses to meet mandatory and optional educational requirements. .

The program content and objectives are illustrated in Table 1.

Table 1

Nursing Education Program

\begin{tabular}{|l|l|}
\hline Content & $\begin{array}{l}\text { Objectives: Upon completion of the } \\
\text { program, nurses will be able to: }\end{array}$ \\
\hline Definition of VAP & Define VAP \\
\hline Etiology of VAP & Explain the etiology of VAP \\
\hline Evidence-based preventative measures & Identify evidence-based preventative \\
(VAP Bundle) & measures (VAP Bundle) \\
\hline Current measures used at Rhode Island & Identify current P\&P at RIH \\
Hospital in the ICU environment & \\
\hline How nurses can impact VAP rates & Describe the impact that can have on VAP \\
& rates \\
\hline
\end{tabular}

Preliminary procedures included the researcher obtaining IRB approval from RIC

and Lifespan. Permission from the Director of Nurses and The Director of the ER was also obtained. Then, during roll call, which happened routinely prior to every shift in the $\mathrm{ER}$, the researcher asked all nurses who worked in critical care to stay for an additional five minutes. At that time, the researcher explained the purpose of the research study and also the procedure that would be used. Nurses were informed that participation was strictly voluntary and that their responses to the pre and post test results would remain anonymous and confidential. The assistant clinical manager also announced the project during the critical care nursing resource meeting.

Copies of the IRB approved informational letter (Appendix D) were left in the breakroom in a designated envelope, attached to copies of the pretest (Appendix E). 
Nurses were asked to carefully review the informational letter, and if they agreed, to then complete the pretest, developed by the researcher. In order to maintain anonymity, nurses were asked to enter their favorite color, two digit birth date and last two numbers of their nursing license on the line labeled ID in the top right corner of the pre and posttest (Example: purple0264). A locked box was placed in the break room and nurses were asked to place the finished pretest in the box. The researcher then collected the lock box after two weeks. Then, the educational poster board regarding VAP was placed in the break room and a VAP study guide was sent via email to all nurses in the ER. The poster board remained displayed for two weeks.

After the poster board was removed, the IRB approved informational letter and the posttest were again placed in the break room. The nurses were reminded to use the same identifier from the pretest on the posttest. The nurses had two weeks to complete the test and then place them in the lock box. The researcher collected the lock box at the end of the two weeks.

Outputs refer to what one would expect after the activity or intervention is completed. In this research project, the expected output was increased knowledge and awareness regarding VAP prevention.

Knowledge was measured with an eight item, multiple-choice exam (Appendix E). This test was adapted from an educational website named Pacific Partners in Innovation (2009). Multiple attempts were made by the researcher to obtain information regarding reliability and validity of the exam to no avail. The company provides educational tools and healthcare products aimed to enhance quality care. Some questions were eliminated secondary to their not meeting the objectives of the provided educational material. The test was reviewed by the CNS in the Emergency Room and was also administered to two nurses who did not participant in the study to ensure clarity of the exam questions. The CNS and the two nurses reported that the questions being asked were clear. In addition 
to answering the pre and posttest, nurses were asked to provide information regarding their years of experience in the critical care area of the emergency room and degree status.

Outcomes/Impact refer to what the researcher expects will occur after the research is conducted. The researcher had three desired outcomes for this research study. The first is directly related to the primary purpose of the study: to increase ER nurses' knowledge base regarding VAP. A long term outcome would be to impact the development of a protocol, or bundle, that includes ventilator-associated pneumonia preventative measures to be initiated in the ER.

The researcher expected that nurses will have increased awareness of the complications of VAP. The project will be presented at the ER practice council meeting and implications for further work including development of a VAP prevention bundle will be discussed. The posterboard developed to educate the ER nurses will be left in the break room to be displayed.

\section{Data Analysis}

Descriptive statistics were used to analyze the study variables. Differences between pre and post scores were evaluated. 


\section{Results}

The target population for this project included approximately 130 nurses who worked in the critical care area of the ER. Out of the possible 130 nurses, 23 nurses (20\%) completed the pretest. Average nursing experience ranged from two years to greater than 30 years, with a mean of 15 . Education levels ranged from ADN to Masters. Four nurses $(15 \%)$ had greater than 30 years of experience; six (25\%) had 5-10 years of experience; two (5\%) had 10-20 years of experience; and four (15\%) had 1-5 years of experience. The remaining seven (40\%) did not provide any information in regard to degree status or years of experience.

Table 2 on the next page illustrates the pretest results by question for each participant as well as an overall percent correct. The pre-test scores ranged from $37.5 \%$ $(n=3)$ to $75 \%$, with a mean score of 57.6 for the pretest. Nine nurses scored a $50 \%$, five received $62.5 \%$, and the remainder $(n=6)$ scored $75 \%$ on the pretest. .

Scores by type of content were analyzed. Questions 1,2 and 4 related to how VAP develops and overall hospital costs per patient and the overall hospital costs per patient for VAP had an overall score of 67\%. Questions 3, 5 through 8 were directed toward VAP preventative measures, which included oral care had overall scores of $73.9 \%$. Questions 6 and 7 were directly to oral care and chlorhexidine use had an average score on the pretest of $40.75 \%$. 
Table 2

Pretest Results by Questions and Overall score $(n=23)$

\begin{tabular}{|c|c|c|c|c|c|c|c|c|c|}
\hline$\underline{\text { ID }}$ & Q \#1 & Q \#2 & Q \#3 & Q\#4 & Q \#5 & Q \#6 & Q \#7 & $\underline{Q} \# 8$ & $\frac{\text { Score } /}{\%}$ \\
\hline 1 & 12.5 & 12.5 & 12.5 & 12.5 & 12.5 & 0 & 0 & 12.5 & $\overline{75.0}$ \\
\hline 2 & 0 & 0 & 12.5 & 0 & 12.5 & 12.5 & 0 & 12.5 & 50.0 \\
\hline 3 & 12.5 & 12.5 & 12.5 & 12.5 & 12.5 & 0 & 0 & 12.5 & 75.0 \\
\hline 4 & 0 & 12.5 & 12.5 & 12.5 & 12.5 & 0 & 12.5 & 0 & 62.5 \\
\hline 5 & 12.5 & 12.5 & 0 & 12.5 & 0 & 0 & 0 & 0 & 37.5 \\
\hline 6 & 0 & 12.5 & 0 & 12.5 & 0 & 0 & 12.5 & 12.5 & 50.0 \\
\hline 7 & 0 & 0 & 12.5 & 12.5 & 12.5 & 12.5 & 0 & 12.5 & 75.0 \\
\hline 8 & 0 & 12.5 & 0 & 12.5 & 0 & 0 & 12.5 & 12.5 & 50.0 \\
\hline 9 & 0 & 12.5 & 0 & 0 & 12.5 & 0 & 0 & 12.5 & 37.5 \\
\hline 10 & 0 & 0 & 0 & 12.5 & 12.5 & 0 & 12.5 & 12.5 & 50.0 \\
\hline 11 & 12.5 & 0 & 12.5 & 12.5 & 12.5 & 12.5 & 0 & 12.5 & 75.0 \\
\hline 12 & 12.5 & 0 & 0 & 12.5 & 12.5 & 12.5 & 12.5 & 12.5 & 75.0 \\
\hline 13 & 12.5 & 0 & 0 & 12.5 & 12.5 & 0 & 0 & 12.5 & 50.0 \\
\hline 14 & 0 & 0 & 12.5 & 12.5 & 12.5 & 0 & 12.5 & 12.5 & 62.5 \\
\hline 15 & 0 & 12.5 & 12.5 & 12.5 & 12.5 & 0 & 0 & 12.5 & 62.5 \\
\hline 16 & 0 & 0 & 12.5 & 12.5 & 12.5 & 0 & 0 & 12.5 & 50.0 \\
\hline 17 & 12.5 & 0 & 0 & 12.5 & 12.5 & 12.5 & 0 & 12.5 & 62.5 \\
\hline 18 & 0 & 12.5 & 0 & 12.5 & 12.5 & 12.5 & 0 & 12.5 & 62.5 \\
\hline 19 & 0 & 0 & 12.5 & 12.5 & 12.5 & 0 & 0 & 12.5 & 50.0 \\
\hline 20 & 0 & 0 & 12.5 & 0 & 12.5 & 12.5 & 0 & 12.5 & 50.0 \\
\hline 21 & 12.5 & 0 & 12.5 & 0 & 12.5 & 0 & 0 & 12.5 & 50.0 \\
\hline 22 & 12.5 & 0 & 12.5 & 12.5 & 12.5 & 12.5 & 0 & 12.5 & 75.0 \\
\hline 23 & 12.5 & 0 & 0 & 0 & 12.5 & 12.5 & 0 & 0 & 37.5 \\
\hline
\end{tabular}

Eight nurses completed the posttest. The average range of nursing experience was 4-15 years with a mean of 10 . Two nurses $(25 \%)$ had $10-15$ years of experience, four $(50 \%)$ had $5-10$ years, and one (12.5\%) had $1-5$ years of experience. The one $(12.5 \%)$ remaining participant did not provide any information regarding degree status or years of experience. The education level ranged from ADN to BSN. Table 3 illustrates the posttest results by question for each participant as well as an overall percent correct. Table 3 
Posttest Results by Question and Overall Score $(N=8)$

\begin{tabular}{|l|l|l|l|l|l|l|l|l|l|}
\hline ID & Q \#1 & Q \#2 & Q \#3 & Q \#4 & Q \#5 & Q \#6 & Q \#7 & Q \#8 & $\begin{array}{l}\text { Score } \\
\%\end{array}$ \\
\hline 1 & 0 & 0 & 12.5 & 12.5 & 12.5 & 0 & 0 & 12.5 & $50 \%$ \\
\hline 2 & 0 & 12.5 & 0 & 12.5 & 12.5 & 0 & 12.5 & 12.5 & $62.5 \%$ \\
\hline 3 & 0 & 12.5 & 12.5 & 12.5 & 12.5 & 12.5 & 12.5 & 12.5 & $87.5 \%$ \\
\hline 4 & 12.5 & 0 & 12.5 & 12.5 & 12.5 & 0 & 0 & 0 & $50 \%$ \\
\hline 5 & 0 & 0 & 0 & 12.5 & 12.5 & 0 & 0 & 0 & $25 \%$ \\
\hline 6 & 12.5 & 12.5 & 12.5 & 0 & 12.5 & 0 & 12.5 & 0 & $62.5 \%$ \\
\hline 7 & 0 & 0 & 12.5 & 0 & 12.5 & 12.5 & 0 & 12.5 & $50 \%$ \\
\hline 8 & 0 & 12.5 & 12.5 & 12.5 & 12.5 & 0 & 0 & 12.5 & $62.5 \%$ \\
\hline
\end{tabular}

The posttest scores ranged from $25 \%$ to $87.5 \%$, with a mean score of $56.3 \%$ for the post-test. Questions related to when and how VAP develops and the overall hospital costs per patient (questions 1, 2 and 4) had an overall score of 62.5\%. Questions 3,5 through 8 were directed toward VAP preventative measures, which included oral care, had an overall score of $70 \%$. Questions 6 and 7 related specifically to oral care and chlorhexidine use had an average score on the posttest of $39 \%$. 


\section{Summary and Conclusions}

Healthcare-associated infections (HAIs) represent a huge financial burden and they contribute significantly to health care costs. The Centers for Disease Control and Prevention (CDC, 2010) defined HAIs as infections that patients acquire during hospitalization while receiving treatment for healthcare conditions. Ventilator associated pneumonia (VAP) is the most common infection in mechanically ventilated patients and the second most common HAI (Sedwick et al., 2012).

Nurses can have a direct impact on prevention of HAIs, including VAP. Lack of current and evidence-based knowledge among nurses regarding preventative measures contributes to the development of HAIs (Gallagher, 2012). The Institute for Healthcare Improvement (IHI) (Gallagher) implemented a ventilator bundle that consisted of several interventions aimed at reducing the incidence of VAP, and various forms of the bundle are used in intensive care units across the country. There is evidence to support that early goal- directed therapy initiated in the ER has a direct impact on patient mortality (ChungEsaki et al., 2011), but there is a lack of research to support that early interventions in the ER can decrease the incidence of VAP. Likewise, education of nurses regarding VAP prevention interventions has been shown to be effective (Blot et al., 2007).

The purpose of this study was to evaluate the impact of an educational program on ER nurses' knowledge of VAP and preventative measures to decrease VAP. At the time of this study, the ER at the study site did not have any VAP prevention measures in place. A pre-, intervention, posttest design was used. The researcher developed an eight question test derived from an educational website Pacific Partners in Innovation (2009). The test questions encompassed information related to VAP such as time to onset of VAP after intubation, costs, and preventative measures that can potentially be implemented in the ER. 
The purpose of the research was explained to the ER critical care nurses' during roll call. The pretests, with the IRB approved informational letters attached individually, were placed in an envelope in the break room. Nurses who were interested were asked to complete the pretest and drop it in a sealed drop box; tests were then collected by the researcher after two weeks. An educational intervention using a posterboard was displayed in the nurses' breakroom for two weeks. The information in the poster board and study guide was derived from the literature review and the investigator's clinical experience, and focused on interventions that could be initiated in the ER. An investigator developed study guide was also dispersed to ER critical care nurses through email. The posttest, with the informational letter attached, was made available after the poster board was removed, using the same procedure as for the pretest. Completed posttests were deposited in a sealed drop box in the break room and collected after two weeks.

A sample of 23 nurses completed the pretest. The findings revealed that ER nurses overall lacked knowledge about VAP, including frequency and procedures for oral care. Pre intervention, $39 \%(n=9)$ of nurses were able to identify that oral care should be performed every four hours and $26 \%(n=6)$ identified the importance of using chlorhexidine every 12 hours when performing oral care. Eighty seven percent $(n=20)$ of the nurses were aware that head of elevation should be thirty degrees or higher for intubated patients.

Eight nurses completed the posttest. Scores regarding oral care were again low: $31 \%(n=2)$ identified that oral care should be performed every four hours and $47 \%(n=3)$ responded that they would use chlorhexidine every 12 hours when performing oral care. One hundred percent $(n=8)$ were aware that head of bed should be thirty degrees or higher for intubated patients. As evidenced by these results, both pre and post, ER 
nurses' were unaware that oral care and the use of chlorhexidine are important interventions to reduce the incidence of developing VAP.

Low participation was a barrier in this project. The researcher's goal was for onethird $(n=30)$ of 130 critical care nurses to participate in the study. Although nurses expressed interest in the topic prior to the start of the research project, there was a lack of participation. Lack of time to complete the intervention and the surveys may have been a contributor to the low participation.

The method used to announce the research project was identified as a limitation. The procedure was for the researcher to pull aside ER critical care nurses after roll call was completed and to then explain the project. Although the researcher attended multiple role calls, it was challenging to find the time to make the announcement. The researcher might have incorporated other ways to increase awareness of the project, such as requesting that the assistant clinical nurse manager make an announcement during role call when the researcher was not present. Sending an email to critical care nurses explaining the project might have increased participation in the project.

Another limitation was the identifier used to administer the pre-posttest. Nurses were asked to place an identifier on the top of the page, which consisted of their favorite color, numerical month of birth, and the last two digits of their nursing license. Many of the nurses verbalized that they did not know their nursing license number. During the posttest, nurses' expressed that they were unable to remember their identifier from the pretest. It is also acknowledged that nurses may not have put an identifier on the tests in order to further limit the ability to be individually identified.

The results indicated that overall posttest scores did not increase after the intervention. It is unclear why this occurred. The intervention was carefully designed from the literature and clinical experience; the use of a poster board as the primary vehicle was determined as a means to allow busy ER nurses flexibility to review the 
material when they were able. Perhaps nurses did not see the value in the project, and did not invest the time needed to complete the intervention and the posttest. Intubated patients are not in the ER for an extended time and nurses had expressed concern in the past that they did not have the time or the materials needed to start the intervention. Although nurses had expressed an interest in the topic, it is possible that they did not see it as directly relevant to them.

In conclusion, this student researcher was able to conclude that further education regarding VAP prevention strategies was needed in the ER. In order to be successful, it will be important prior to further program development that interdisciplinary support be identified and secured. A comprehensive needs assessment is recommended, and involvement of the nurses and interdisciplinary team within the ER. Further research is needed to measure VAP rates after initiation of a modified VAP bundle beginning in the emergency room.

Next, recommendations and implications for advanced practice will be presented. 


\section{Recommendations and Implications for Advanced Practice}

Healthcare-associated infections (HAIs) are a source of increased material and human cost in the US, and evidence-based guidelines and protocols have been developed in the attempt to decrease the incidence of HAIs. The Joint Commission requires hospitals to demonstrate that they have preventative guidelines in place to reduce HAIs. Currently, most VAP bundles are directed toward intensive care units and do not address early intervention measures to begin immediately after intubation occurring outside of intensive care. Most components of the ventilator bundle can be implemented and managed by nurses. A role of the APRN is to work with nursing colleagues to ensure that they have the knowledge needed to implement best practices. Increasing use of technology such as online learning can be beneficial in implementing educational interventions. Many institutions have mandatory, yearly competencies that are completed online and afford the institution the ability to track completion. Educational interventions have been shown to be effective in increasing nurses' knowledge; on-going support and assessment is needed to evaluate compliance and determine the impact on patient outcomes. Education alone is not sufficient to initiate and sustain a practice change. Ventilator associated pneumonia prevention is primarily dependent upon nursing interventions; nurses need to understand the importance of preventative strategies and consistently implement them into practice. This might best be accomplished by using change principles within whatever interventions are planned, and by providing continuing support and reinforcement.

At the institutional level, the APRN can be essential in developing and implementing an evidence-based, modified VAP bundle to begin in the ER. Early goaldirected therapy has been shown to be beneficial in improving patient outcomes and decreasing hospital costs. In order to accomplish this goal, the APRN would potentially assume a leadership role within the interdisciplinary team to further develop, implement, 
and evaluate an evidence-based, modified bundle. Development of an interdisciplinary VAP task force in the ER would be useful in assuring institutional support for the initiative, piloting the intervention in the ER, and evaluating the impact by tracking VAP rates. If the intervention was shown to decrease VAP rates, the APRN could be instrumental in formally implementing this practice change.

Further work is needed at the national level to influence the development of VAP preventative guidelines specific to the ER. Advanced practice nurses who are members of the Emergency Nurses Association (ENA) and other national bodies can be influential in lobbying for support for these initiatives. Health care organizations such as IHI have been instrumental in moving VAP initiatives forward; given that a significant number of hospitalized patients are admitted through the ER, extending the focus of preventative interventions to the ER is an important pursuit.

Focus on decreasing health-care costs while maintaining patient safety is the goal of many organizations such as The Joint Commission, Medicare and Medicaid, and the Agency for Healthcare Research and Quality. The Center for Medicare and Medicaid Services (CMS) is no longer reimbursing hospitals for certain HAIs, including VAP (Ryan, 2012). Reimbursement is focused on value based purchasing, which includes such factors as patient satisfaction and patient outcomes (Ryan). Research has shown that early goal directed therapy that begins in the ER has decreased patient mortality, but minimal literature exists regarding VAP prevention initiated in the ER. It is important to note that the literature also supports that increased time in the ER increases a patient's likelihood of developing VAP. While it would be challenging to reduce the length of time that an intubated patient remains in the ER, this could be an area for future research. Further research related to VAP prevention strategies initiated in the ER is indicated. Study with larger samples, a diversity of subjects, and testing a cadre of potential strategies is indicated. The ENA has been a leader in the development of standards for ER practice. 
The ENA can likewise be a driver and potential funder of further VAP prevention research in the ER. 


\section{References}

Amin, A. (2009). Clinical and economic consequences of ventilator-associated pneumonia [Supplemental Material]. Clinical Infectious Disease, S36-S43. http://dx.doi.org/10.1086/599814

Blot, S. I., Labeau, S., Vandijck, D., Van Aken, P., \& Claes, B. (2007, June 1). Evidencebased guidelines for the prevention of ventilator-associated pneumonia: results of a knowledge test among intensive care nurses. Intensive Care Med, 33, 14631467. http://dx.doi.org/10.1007/s00134-007-0705-0

Bonten, M. J. (2011). Ventilator-associated pneumonia: preventing the inevitable. Clinical Infectious Disease, 52, 115-121. http://dx.doi.org/10.1093/cid/ciq075

Carr, B. G., Kaye, A. J., Wiebe, D. J., Gracias, V. H., Schwab, W., \& Reilly, P. M. (2007). Emergency department length of stay: A major risk factor for pneumonia in intubated blunt trauma patients. The Journal of Trauma, 63(1), 9-12. http://dx.doi.org/10.1097/TA.0b013e31805d8f6b

Chung-Esaki, H. M., Wilson, J. G., \& Rodriguez, R. M. (2011). Caring for the critically ill: A continuum from the emergency department to the intensive care unit. ICU Director, 2, 141-146. http://dx.doi.org/10.1177/19444516111423099

Gallagher, J. (2012, May). Implementation of ventilator-associated pneumonia clinical guideline (Bundle). The Journal for Nurse Practitioners, 8, 377-382.

Gatell, M. J., Roig, M. S., Vian, O. H., Santin, E. C., Duaso, C. T., Moreno, I. F., \& Daunis, J. V. (2012). Assessment of a training programme for the prevention of ventilator-associated pneumonia. Nursing in Critical Care, 17, 285-292. http://dx.doi.org/10.1111/j.1478-5153.2012.00526.x

Grap, M., Munro, C. L., Unoki, T., Hamilton, A., \& Ward, K. (2012). Ventilatorassociated pneumonia: The potential critical role of emergency medicine in 
prevention. Journal of Emergency Medicine, 42, 353-362. Retrieved from www.medscape.com

Grgurich, P. E., Hudcova, J., Lei, Y., Sarwar, A., \& Craven, D. E. (2013, April). Diagnosis of ventilator-associated pneumonia: controversies and working toward a gold standard. Current Opinion in Infectious Diseases, 26, 140-150. http://dx.doi.org/10.1097/QCO.0b013e32835ebbd0

Healthcare Associated Infections. (2010). Retrieved June 28, 2013, from www .cdc.gov Kellogg Foundation. Logic model of program development. . Retrieved from http://www.wkkf.org/Pubs/Tools/Evaluation/Pub3669.pdf

Knowles, M. S., Holton, E. F., \& Swanson, R. A. (2005). The Adult learner:The definitive Classic in Adult Education and Human Resource Development (6th ed.). Burlington, MA: Elsevier.

Labeau, S., Vandijck, D., Rello, J., Adam, S., Rosa, A., Wenisch, C., ... Blot, S. (2008). Evidence-based guidelines for the prevention of ventilator-associated pneumonia: results of a knowledge test among European intensive care nurses. Journal of Hospital Infection, 70, 180-185. http://dx.doi.org/10.1016/j.jhin.2008.06.027

Mateo, M. A., \& Kirchhoff, K. T. (2009). Research for Advanced Practice Nurses. New York: Springer Publishing Company.

Meherali, S. M., Parpio, Y., Ali, T. S., \& Javed, F. (2011). Nurses' knowledge of evidence-based guidelines for prevention of ventilator associated pneumonia in critical care areas: A pre and post test design. Journal Ayub Medicine Coll, 23, 146-149. Retrieved from http://www.ayubmed.edu.pk/JAMC/201-1/Salima.pdf Mietto, C., Pinciroli, R., Patel, N., \& Berra, L. (2013, June 1). Ventilator associated pneumonia: evolving definitions and preventative strategies. Respiratory Care Journal, 58, 990-1007. http://dx.doi.org/10.4187/respcare.02380 
Novosel, T. J., Hodge, L. A., Weireter, L. J., Britt, R. C., Collins, J. N., Reed, S. F., \& Britt, L. (2012). Ventilator-associated pneumonia: depends on your definition. The American Surgeon 78. 851-854. Retrieved from www.proquest.com

Pacific Partners in Innovation Education Series. (2009). Pacific Partners in Innovation Education Series: Ventilator Associated Pneumonia. www.pacificpartners.biz Rea-Neto, A., Youssef, N. C., Tuche, F., Brunkhorst, F., Ranieri, V., Reinhart, K., \& Sakr, Y. (2008, April 21). Diagnosis of ventilator-associated pneumonia: a systematic review of literature. Critical Care, 12 . http://dx.doi.org/10.1186/cc6877

Ryan, J. (2012, September). Leveraging the skills of APRNs to prevent HAIs. Nursing Mangement, 43. http://dx.doi.org/10.1097/01.NUMA.0000418774.23315.70

Scott, R. (2009). The direct medical costs of healthcare-associated infections in U.S hospitals and the benefits of prevention. Retrieved from www.cdc.gov

Sedwick, M., Lance-Smith, M., Reeder, S., \& Nardi, J. (2012, August, 2012). Using evidence-based practice to prevent ventilator-associated pneumonia. Critical Care Nurse 32(4), 41-51. 
Appendix A
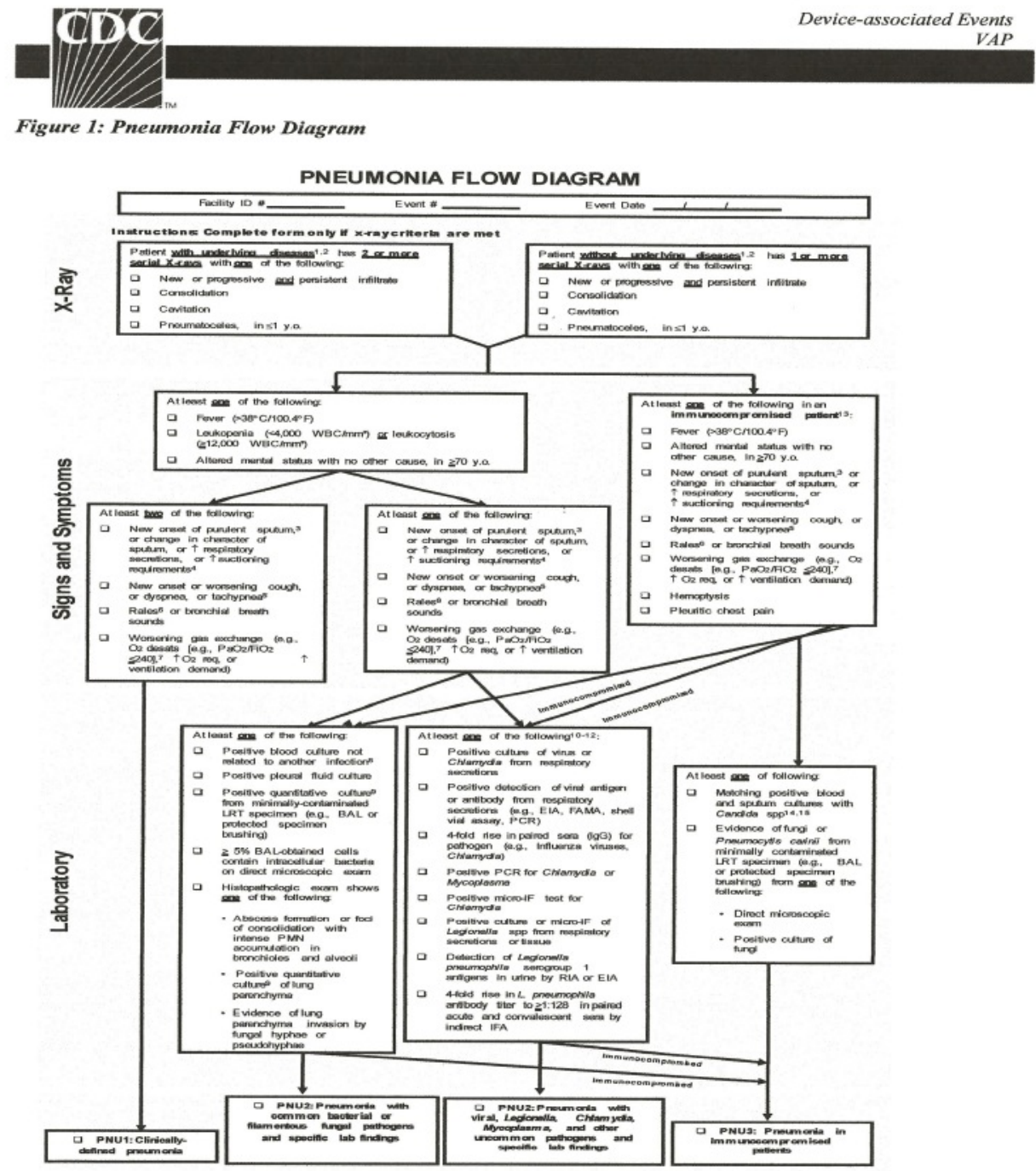

January 2013

6-10 


\section{Appendix B}

Program: Preventing Ventilator Associated Pneumonia: Educating Emergency Room Nurses

Logic Model

Situation: Ventilator associated pneumonia (VAP) is the second most common hospital acquired infection. Intubation and mechanical ventilation increase a patient's risk of developing VAP. VAP is considered to be a "preventable disease". Current VAP measures are geared toward the Intensive care unit. Little literature exists regarding the emergency room and VAP.

\begin{tabular}{|c|c|c|c|c|c|}
\hline $\begin{array}{l}\text { Inputs } \\
\text { CNS support }\end{array}$ & \multicolumn{2}{|l|}{$\begin{array}{l}\text { Outputs } \\
\text { Activities }\end{array}$} & $\begin{array}{l}\text { Outcomes - Impact } \\
\text { Short }\end{array}$ & \multicolumn{2}{|l|}{ Medium } \\
\hline $\begin{array}{l}\text { CNS support } \\
\text { Director of Infection } \\
\text { Control input and } \\
\text { support } \\
\text { Staff interest in } \\
\text { topic } \\
\text { Focused needs } \\
\text { assessment } \\
\text { Limited literature/ } \\
\text { research re: VAP } \\
\text { prevention in EDs } \\
\text { Pre-post test } \\
\text { identified } \\
\text { Voluntary } \\
\text { recruitment }\end{array}$ & $\begin{array}{l}\text { Evidence based } \\
\text { poster and study } \\
\text { guide developed, } \\
\text { based on literature, } \\
\text { clinical experience, } \\
\text { and consideration } \\
\text { of current RIH } \\
\text { policy and } \\
\text { procedure. } \\
\text { Incorporated } \\
\text { Knowles' adult } \\
\text { learning principles } \\
\text { IRB approval } \\
\text { obtained } \\
\text { Pre and post test } \\
\text { administered }\end{array}$ & $\begin{array}{l}\text { All nurses working } \\
\text { in the critical care } \\
\text { area of the } \\
\text { emergency room } \\
\text { were invited to } \\
\text { voluntarily } \\
\text { participate }\end{array}$ & $\begin{array}{l}\text { Increased } \\
\text { knowledge of VAP } \\
\text { This involves } \\
\text { learning as a result } \\
\text { of the educational } \\
\text { intervention }\end{array}$ & $\begin{array}{l}\text { Nurses will } \\
\text { incorporate HOB } \\
\text { elevation and } \\
\text { mouth care in care } \\
\text { of ventilated } \\
\text { patients in the } \\
\text { emergency room }\end{array}$ & $\begin{array}{l}\text { Potentially impact } \\
\text { the development of } \\
\text { a VAP prevention } \\
\text { bundle/ protocol }\end{array}$ \\
\hline \multicolumn{3}{|c|}{$\begin{array}{l}\text { Assumptions: Staff will be willing to participate, CNS and } \\
\text { Director of infectious disease support, Staff willingness to learn }\end{array}$} & \multicolumn{3}{|c|}{$\begin{array}{l}\text { External Factors: Staff resistance, Staff unwillingness to learn or } \\
\text { particpate in anonymous pre-post test. Staff completing pre or } \\
\text { post test but not both. }\end{array}$} \\
\hline
\end{tabular}


Appendix C

Ventilator Associated Pneumonia

Study Guide

Ventilator associated pneumonia (VAP) is a lower respiratory tract infection that develops in patients that are intubated for greater than 24 hours (Sedwick, Lance-Smith, Reeder, \& Nardi, 2012). VAP is the most common infection in ventilated patients and the second most common hospital associated infection. Mortality rates associated with VAP range between 20\% and 70\% (Meitto, Pincoroli, Patel, \& Burra, 2013). The Center for Medicare and Medicaid listed VAP as one of the "reasonably preventable diseases" leading to increased morbidity, mortality, and health care costs (Gallagher 2012). The average hospital cost to care for a patient that develops VAP is approximately $\$ 40,000$ per patient (Gallagher, 2012). Reduction of VAP is a national patient safety goal (Mietto, et al, 2013).

The presence of an endotracheal tube (ETT) decreases the body's natural ability to respond to infectious processes, which is to cough or eliciting a gag reflex (Mietto, et al, 2013). When an ETT is present secretions settle around the posterior pharynx and eventually are aspirated (Sedwick, et al, 2012). Pseudomonas and Acinetobacter are two highly infectious pathogens associated with increased mortality among intubated patients (Amin, 2009).

The Institute of Healthcare Improvement developed a ventilator bundle that consists of evidence- based interventions to decrease the incidence of VAP. The interventions include HOB elevation 30 degrees or higher, DVT and PUD prophylaxis, oral care with chlorhexadine, sedation vacation, and weaning assessment (Gallagher, 2012). Many healthcare institutions across the country have adopted some form of the ventilator 
bundle. The Joint Commission requires hospitals to prove they have preventative guidelines in place to reduce hospital acquired infections (Gallagher, 2012). Rhode Island Hospital has a VAP bundle that incorporates these interventions. RIH bundle consists of weaning, HOB elevation, sedation vacation, PUD prophylaxis, DVT prophylaxis, daily glucose, mouth care and most important proper handwashing technique.

There is a lack of studies regarding these interventions beginning in the emergency room. One article by Grap et al (2012) identified the need of early VAP intervention to begin in the emergency room. Focused interventions that can be initiated int the emergency room included head of bed elevation to decrease aspiration, oral care with the use of chlorhexidine (gel or mouth rinse), management of endotracheal cuff pressure, subglottic suctioning, and ulcer prophylaxis. (Chung- Esaki, Wilson, \& Rodriguez, 2011). Unfortunately there is no research to support if early interventions in the emergency room decrease the incidence of VAP. 
Appendix D

IRB Approval: $\quad$ 1/27/2014

Informational Letter

IRB Accepted 1/27/2014

IRB Expiration: 1/26/2015

You are being asked to take part in a research study called Proventing Ventilator

Arsociatod Pheumonia: Educating Emengency Room Nurses; the purpose of the study is to measure emergency room uurses' kmowledge regarding ventilator associated

paeumonia and the effects of an echcational intervention on that knowledge. Dans

Ferrazzano, a student at Rhode Island College, is conducting this study in conjunction

with the Principal Investigator, Cynthia Padula

If you agree to participate, you will be asked to complete an eight question pre-test. You will then be asked to review a poster display board that includes information about ventilator- associated pneumonia and a study guide that will be sent to you via lifespan email. After reviewing these items, you will be again asked to complete a post-test. The total time to complete these activities is about 20 minutes.

There are no questions that sbould cause you discomfort and there are no identified risks to participating. Your taking part in this research project is completely volumtary. If you do not want to participate, you are free to choose not to and may withdraw your participation at any time.

Your participatioa may increase your knowledge regarding ventilator associated poeumoaia. Neitber the pre nor post test will include identifiable information; bowever it may be possible to identify you based on your responses to the questions. When you fill out the test, please use your favorite color, month of birth and last two digits of nursing license (example: Purple0211) as your identifier, also include your degree status and years of experience. When you bave completed the test, please place it in the locked box 
in the break-room that has been provided. The Researcher and Principal Investigator will be the only individuals that will have access to the locked box. Neither the pre nor post test will include information, however it may be possible to identify you based on your responses to the questions.

You may ask any questions you have now. If you have any questions later, you may contact Dana Ferrazzano at 480-5713, or at dferrazzano@ lifespan.org. You may also contact the faculty mentor, Cynthia Padula, $\mathrm{PhD}, \mathrm{RN}$ at $456-9720$ or cpadula@ric.edu. If you think you were treated unfairly or would like to talk to someone other than the researcher about your rights or safety as a research participant, please contact $\mathrm{Dr}$. Christine Marco, Chair of the Rhode Island College Institutional Review Board at IRB a ric.edu, or by phone at 401-456-8598, or by writing to Christine Marco, Chair IRB; c/o Department of Psychology, Horace Mann Hall 311; Rhode Island College; 600 Mount Pleasant Avenue; Providence, RI 02908.

If you have any further questions about your rights as a research subject please feel free to call Patricia Houser, in the Lifespan Office of Research Administration, at 444-2099.

Thank you very much for you time!

Dana Ferrazzano RN, BSN, CCRN

Rhode Island Hospital 
Appendix E

Identifier

Degree

Years of experience

\section{VAP Pre and Post Test}

1. Ventilator Associated Pneumonia (VAP) is defined as a lower respiratory tract infection developed in patients who have been intubated and mechanically ventilated for greater than
a. 24 hours
b. 48 hours
c. 8 hours
d. 72 hours

2. On average, a case of VAP can potentially add about how much money to typical hospital costs per patient?
a. $\$ 1,000$
b. $\$ 12,000$
c. $\$ 100,00$
d. $\$ 40,000$

3. Which of the following is NOT included in the guidelines for prevention of VAP?

a. Elevation of the head of the bed to 30 degrees or greater during mechanical ventilation

b. Hand washing before and after contact with the patient

c. Frequent sedation of patients while on mechanical ventilation to avoid coughing and aspiration

d. Vigorous oral hygiene

4. Patients develop VAP from

a. oral or Gastric pathogens that migrate into the lungs

b. poor cleaned ventilators

c. visiting family members

d. failure to be intubated properly

5. While being mechanically ventilated, and if not contraindicated, it is recommended that patients be elevated to
a. 20 degrees or less
b. 30 degrees or greater
c. 50 degrees or less
d. 90 degrees or greater

6. How often should mouthcare be performed on an intubated patient?

a. every 24 hours

b. every 8 hours 
c. every 4 hours

7. Based on evidence based guidelines, how often should mouth care be performed with chlorhexidine?
a. Every 2 hours
b. every 24 hours
c. every 4 hours
d. every 12 hours

8. How often should suctioning be performed on an intubated patient?
a. as needed
b. every time mouth care is performed
c. when there are secretions present
d. all of the above 